\title{
Ultrastructural and EDS Study of Sialoliths of the Salivary Glands
}

\author{
A.P. Alves de Matos*, P. A. Carvalho**, A. Almeida*, L. Duarte***, R. Vilar** and J. Leitão* \\ *Departamento de Biomateriais, Faculdade de Medicina Dentária, Universidade de Lisboa, 1649- \\ 003 Lisbon, Portugal. \\ **Departamento de Engenharia de Materiais, Instituto Superior Técnico, Universidade Técnica de \\ Lisboa, Av. Rovisco Pais, 1049-001 Lisboa, Portugal. \\ ***Serviço de Cirurgia Maxilo-Facial, Hospital de S. José, R. António Serrano, 1100 Lisboa, \\ Portugal.
}

Sialoliths, frequently developing in de ducts of salivary glands, are a cause of pain, inflammation and infection of the affected glands. Ultrastructural studies combined with EDS analysis have revealed variable patterns of structural elements with variable calcification levels. However the mechanism of formation of these complex stones and the cause of their formation are still unclear $[1,2]$.

We have studied 8 sialoliths from the parotid, submandibular and submaxilar glands by scanning electron microscopy combined with EDS analysis. Sialoliths were broken approximately through the middle, mounted in carbon supports with carbon painting, and covered with a carbon layer in a rotary vacuum evaporator. Selected samples were embedded in an epoxy resin, grounded with $\mathrm{SiC}$ paper and polished using alumina suspensions.

Smaller sialoliths usually displayed multiple thin (1-5 $\mu \mathrm{m})$ concentric layers surrounding an compact core (figs. 1-2), while larger samples displayed multiple cores and in one case lacked the thin layer component (Fig. 3). The layers displayed variable fine structure from homogeneous to

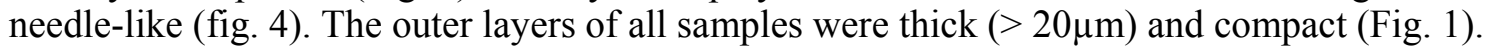
Polished samples revealed structures that were not clearly identified in fractured ones. Among them, droplet-like structures in different stages of calcification were a prominent feature of some samples (fig 5). The variable structures observed suggest that several growth mechanisms may be present, probably related to the disease process.

EDS analysis showed that the central amorphous core were always heavily mineralized and composed of phosphorus and calcium (fig. 6), probably representing hydroxyapatite [2]. Thin layers were heavily mineralized like the core or composed by a sulphur rich substance (fig. 7). Sulphurcontaining areas with variable content of calcium and phosphate were also detected in the outer layers of large samples and were darker in backscattered electron images than mineralized areas (fig 3). $\mathrm{Mg}, \mathrm{Cl}$, and $\mathrm{Si}$ were also detected in variable amounts in the sialoliths. The sulphur-containing areas may represent organic substances with high $\mathrm{S}$ content [3], but not all of them seem to be undergoing a calcification process.

\section{References}

[1] F. Grases et al., Clin. Chem. Acta 334 (2003) 131-136.

[2] N. Tanaka et al., Med. Electron Microsc. 36 (2003) 120-126.

[3] J.M. Riesco et al., J. Oral Pathol. 28 (1999) 451-455. 

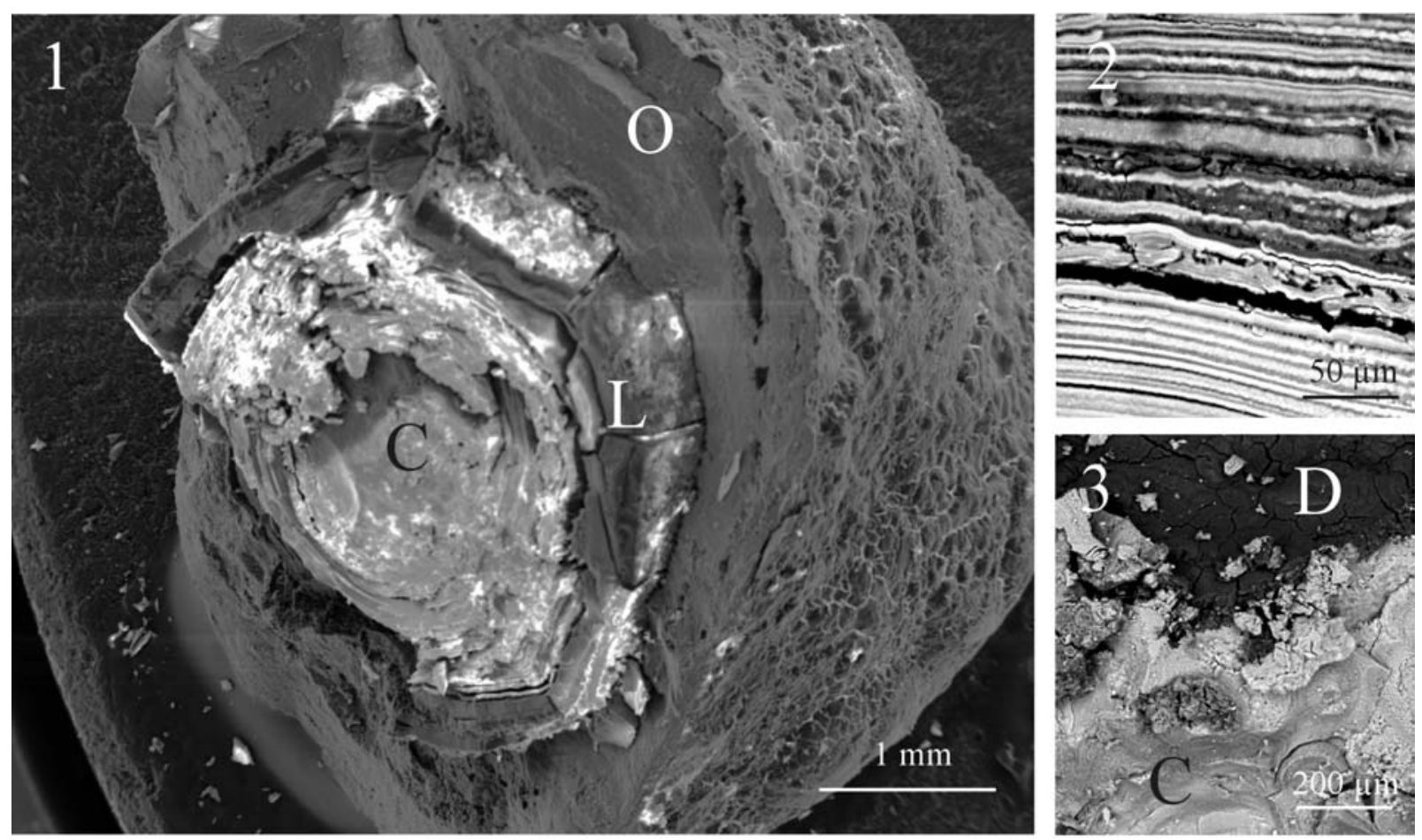
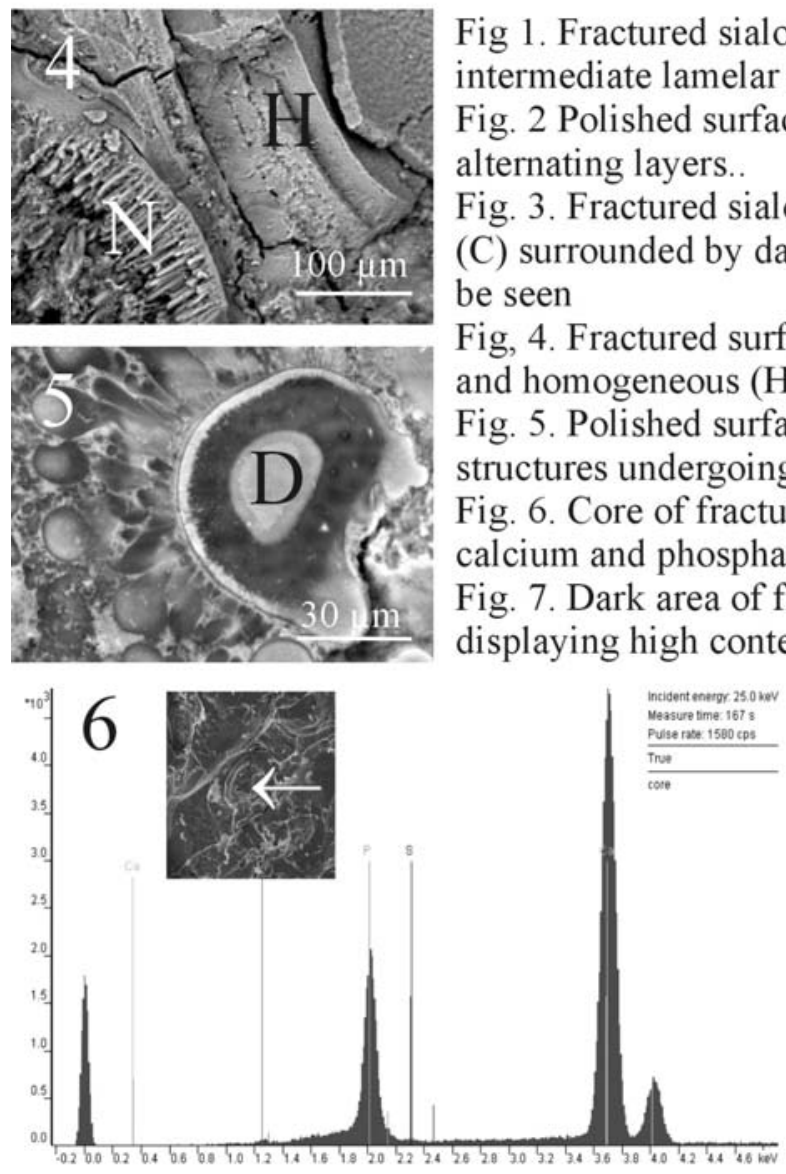
alternating layers. be seen
Fig 1. Fractured sialolith. Secondary electron image. Core (C), intermediate lamelar area (L) and outer thick layers $(\mathrm{O})$.

Fig. 2 Polished surface. Backscatered electron image. Dark and clear

Fig. 3. Fractured sialolith. Backscattered electron image. Calcifyed core (C) surrounded by dark sulfur-rich matrix (D). No layered structure can

Fig, 4. Fractured surface. Secondary electron image. Needle-like (N) and homogeneous $(\mathrm{H})$ layers.

Fig. 5. Polished surface. Backscattered electron image. Droplet like structures undergoing calcification (D).

Fig. 6. Core of fractured sialolyth. EDS graph. The matrix contains calcium and phosphate (Arrow - analysis spot).

Fig. 7. Dark area of fractured sialolith in backscattered electron image displaying high content of sulfur. EDS graph (Arrow - analysis spot).

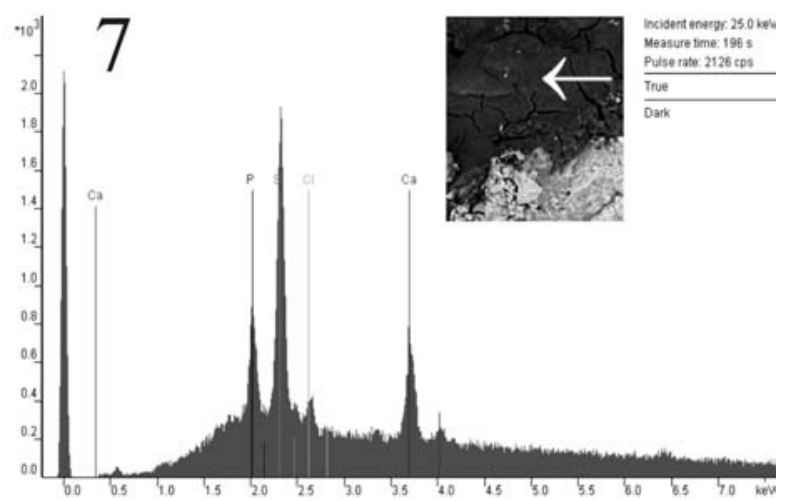

